\title{
Quantum phase transition: Van Vleck antiferromagnet in a magnetic field
}

\author{
I.M.Ivanova ${ }^{1}$, V.M.Kalita ${ }^{2}$, V.O.Pashkov ${ }^{3}$, V.M.Loktev ${ }^{4}$ \\ ${ }^{1}$ National Technical University of Ukraine "KPI", Prosp. Pobedy 37, Kyiv 03056, Ukraine \\ 2 Institute of Physics of the National Academy of Science of Ukraine, Prosp. Nauki 46, Kyiv 03680, Ukraine \\ 3 National Aviation University, Kosmonavta Komarova Ave. 1, Kyiv 03058, Ukraine \\ ${ }^{4}$ Bogolyubov Institute for Theoretical Physics of the National Academy of Science of Ukraine, Metrolohichna \\ Str. 14, Kyiv 03680, Ukraine
}

Received May 7, 2008, in final form July 7, 2008

\begin{abstract}
Theoretical description of magnetic properties of antiferromagnetic state, induced by the longitudinal magnetic field, in the Van Vleck - singlet - magnet with single-ion anisotropy of "easy-plane" type and ion spin $S=1$ is proposed. It is shown that the quantum phase transition to the antiferromagnetic state is connected with the spontaneous appearance of spin polarization in the easy plane. Spin polarization of the ground nondegenerated state proves to be the order parameter of such a transition and the Landau thermodynamic approach can be employed for its (transition) description. The magnetic properties which include the field behavior of magnetization and magnetic susceptibility of the antiferromagnetic phase in the fields of different directions are studied. An attempt is made to qualitatively compare the obtained results with the available experimental data.
\end{abstract}

Key words: quantum magnetic phase transition, single-ion anisotropy, spin polarization, Van-Vleck antiferromagnet

PACS: $75.10 .-b, 75.10 . J m, 75.30 . G w, 75.30 . K z, 75.50 . E e$

\section{Introduction}

Quantum phase transition is the transition which occurs at temperature $T=0$, when some external "governing" parameter is changed. As a rule, these are the transitions of "order-order" type, which corresponds to the zero entropy of both phases. Transition point is called quantum transition point [1] and now there are many examples of such transitions. It will be shown that one of the interesting cases of the quantum phase transitions in the Van Vleck antiferromagnets is the magnetic transition in the external field.

Indeed, it is a well-known fact that magnetization of classical (or, which is the same, weakly anisotropic) antiferromagnets (AFMs) at low temperatures (far below the Neel one $T_{\mathrm{N}}$ ) is connected only with sublattice magnetization turn [2]. From this fact, it is usually supposed that their magnitude remains constant, and only their directions change under the effect of the external magnetic field. The character and peculiarities of magnetization process (spin-flip, spin-flop as well as orientation phase transitions of the Ist order) of such AFMs depend on the following parameters: value and direction of the external magnetic field, anisotropy, and intersublattice exchange [3-6]. For example, field behavior of magnetization in "easy-plane" two-sublattice dihalogenids $\mathrm{NiCl}_{2}$ or $\mathrm{CoCl}_{2}$ of iron group [7-11] is well satisfied by quasi-classical approach, although these magnets are markedly different. The "easy-plane" single-ion anisotropy (SIA) in $\mathrm{NiCl}_{2}$ is much lower than the exchange - each ion orbital moment in crystal field is almost completely frozen. At the same time, there is only partial freezing of the orbital moment in $\mathrm{CoCl}_{2}$, and "easy-plane" SIA is approximately half the exchange (in order of its magnitude [8]). Field dependence of the induced magnetostriction in these AFMs [12-14] also agrees with a conception of the tilt of sublattice magnetizations, keeping the absolute value. 
However, there is a family of crystals among AFMs, where SIA exceeds the inter-ion exchange $[15,16]$. These are the so-called Van Vleck, or singlet antiferromagnets (SAFMs). They have no magnetic ordering at any temperature, up to $T=0$. Such materials include, in particular, hexagonal crystals of $A B X_{3}$ type, where $A$ is the ion of alkali metal $(A=\mathrm{Cs}, \mathrm{Rb}), B$ is the transition metal $(B=\mathrm{Fe}), X$ is the halogenyde $(X=\mathrm{Cl}, \mathrm{Br})$. In these crystals, magnetic moments induced by external field on paramagnetic ions $B^{2+}$, form, on the one hand, antiferromagnetic chains along $C_{3}$ axis, and, on the other hand, triangular structures in basic plane (see reviews [1,1719]). There are also some other compounds classed with SAFMs, including the so-called DTN, the chemical formula of which is $\mathrm{NiCl}_{2}-4 \mathrm{SC}\left(\mathrm{NH}_{2}\right)_{2}$ [20-23]. It also has (AFM) chains Ni-Cl-Cl-Ni along a "hard" magnetic axis, although the mean spin on each site is equal to zero at field absence, since the parameters of both intra- and intersublattice exchange are exceeded by SIA. It should be emphasized that DTN can be considered to belong to a group of two-sublattice SAFMs, which have a crystal structure other than that of $\mathrm{NiCl}_{2}$, and another character of exchange interactions, which are much lower in the value than the single-ion anisotropy $[22,23]$.

The magnetization process in SAFMs is fundamentally different from that taking place in classical Neel AFMs [25-28]. Firstly, they have no magnetic ordering at the absence of the external magnetic field. Hence, they have no magnetic sublattices. Secondly, magnetic, or in particular AFM, ordering in SAFMs may appear spontaneously by way of the quantum (in definition of reference [24]) phase transition induced by the magnetic field [1,17-23].

The weak dependence of magnetic susceptibility on external magnetic field, thus, represents some sort of peculiarity of AFM phase. As a result, the observed magnetization actually follows the linear field behavior $[22,23,29,30]$. In other words, this behavior of magnetization of the system (but not the proper sublattice magnetization) in the AFM phase turns out to be similar to the magnetization induced by the external field in Neel AFMs. This could be understandable if the transition to the AFM phase were a phase transition of the Ist order. Then, at the transition point, the sublattices could magnetize, due to jump (in the presence of corresponding susceptibility singularity), and with further field growth the sublattice magnetic vectors would turn only. However, the experiment shows that transformation of the non-magnetic (singlet) state into the AFM state takes place continuously, i.e., this magnetic transformation is a phase transition of the IInd order $[22,23,29,30]$. The latter demonstrates that sublattice magnetizations appear and change from their initial zero value to maximal value also continuously. Therefore, the classical approach with the constant module of average sublattice spin for SAFMs is not applicable fundamentally.

As it follows from the above, there still remain some unresolved issues in the description of induced magnetic phase transition into the AFM phase, and its magnetic characteristics (field dependencies of sublattice and of the system as a whole, magnetization, magnetic susceptibility, magnetostriction) in Van Vleck systems.

Below we will proceed from an assumption that in such magnets the intrinsic spontaneous magnetic (or AFM) moment is equal to zero. So, without the external magnetic field, they lack the magnetic ordering temperature. Seemingly, the fact that the magnetic (dipole) moment or, in other words, magnetization (spin) on the site is equal to zero is indicative not only of the absence of any magnetic ordering, but, most probably, of any magnetic contributions to physical properties of the corresponding systems. However, this is not the case, because the absence of ordinary exchange-induced - spin ordering does not exclude the presence of the ordering of another type, e. g., the quadrupole one. The latter, in one or the other way, is characteristic of all SAFMs, which, in turn, are a special case in the magnetic crystals with more specific - nematic - type of spin ordering $^{1}[31]$.

It should be noted that some studies (e. g. [32-35]), describe the phase transition between the singlet and induced AFM states by using the representation of Bose-Einstein condensation of magnons. Indeed, the appearance of magnetization in finite magnetic fields can be formally described in terms of some magnetic excitations condensation. But in reality no true condensation

\footnotetext{
${ }^{1}$ As pointed in the recent study [31], the nematic (in the vary sense of this term) ordering in strongly anisotropic magnets is absent. However, the ground state of such magnet, that is singlet, does not differ, in physical sense, from the previous one which is caused by the large biquadratic exchange $[27,28]$.
} 
of quasiparticles occurs in the observed systems, because, and it will be shown below, one should consider rearrangement of the ground state only, and, hence, virtual, rather than real magnons [36].

Below we consider the model of strongly anisotropic, two-sublattice AFM with ion bare spin $S=1$. In the framework of quantum approach an attempt will be made to describe the crystal magnetization, magnetic susceptibility and magnetostriction at magnetically induced phase transition from the initial singlet state to the spin-ordered state. To calculate physical characteristics of the system, we will use the total energy $E$, that is the sum of relevant contributions:

$$
E=E_{\mathrm{exch}}+E_{\mathrm{an}}+E_{\mathrm{h}}
$$

where $E_{\text {exch }}$ is the exchange energy; $E_{\text {an }}$ is the magnetic anisotropy energy and $E_{\mathrm{h}}$ is Zeeman energy.

This work is dedicated to prof. I.V. Stasyuk, whose research of the diagram methods of calculation for Hubbard operators helped in understanding the magnetic properties of magnets with strong SIA.

\section{Ground state of the model}

According to above-said, for simplicity let us limit our consideration to the bilinear anisotropic (intra- and intersublattice) exchange interaction, single-ion "easy-plane" anisotropy and Zeeman contribution. In this case, the simplest model Hamiltonian of a system, which defines the first three contributions, $E_{\text {exch }}, E_{\text {an }}$ and $E_{\mathrm{h}}$, in equation (1), can be written down as follows:

$$
H=\frac{1}{2} \sum_{\mathbf{n}_{\alpha}, \mathbf{m}_{\beta}} J_{\mathbf{n}_{\alpha} \mathbf{m}_{\beta}} \mathbf{S}_{\mathbf{n}_{\alpha}} \mathbf{S}_{\mathbf{m}_{\beta}}+\frac{1}{2} \sum_{\mathbf{n}_{\alpha}, \mathbf{m}_{\beta}} J_{\mathbf{n}_{\alpha} \mathbf{m}_{\beta}}^{Z} S_{\mathbf{n}_{\alpha}}^{Z} S_{\mathbf{m}_{\beta}}^{Z}+D \sum_{\mathbf{n}_{\alpha}}\left(S_{\mathbf{n}_{\alpha}}^{Z}\right)^{2}-\mathbf{h} \sum_{\mathbf{n}_{\alpha}} \mathbf{S}_{\mathbf{n}_{\alpha}},
$$

where $\alpha, \beta=1,2$ are the magnetic sublattice indices, the numbers of which in the considered system was chosen to be equal to 2 ; vectors $\mathbf{n}$ and $\mathbf{m}$ specify the spins position in magnetic sublattices described by spin operators $\mathbf{S}_{\mathbf{n} \alpha}$; constant $D>0$ that reflects an "easy-plane" magnetic structure; magnetic field $\mathbf{h}$ is determined in energy units, hence $\mathbf{h}=\mu_{\mathrm{B}} g \mathbf{H} ; \mathbf{H}$ is the magnetic field. Crystallographic symmetry axis $O Z$ is perpendicular to the "easy" plane. It is at $\mathbf{h} \| O Z$ that the magnetic field induces the phase transition to the AFM state. Transverse field $\mathbf{h} \perp O Z$ in two-sublattice SAFM does not induce any phase transitions. Parameter $J_{\mathbf{n}_{\alpha}} \mathbf{m}_{\beta}$ characterizes the value of an isotropic part of the exchange interaction, and $J_{\mathbf{n}_{\alpha} \mathbf{m}_{\beta}}^{Z}$ characterizes the exchange anisotropy, which, in principle, can be of either "easy-axis" or "easy-plane" type. However, we will assume that the inter-ion anisotropy, like SIA, relates to the same, i.e "easy-plane", type.

The convenience of these restrictions is conditioned by the fact that in such a situation both sublattices become symmetric relatively to the external field, what allows to reduce twice the number of equations.

Analysis of possible eigenstates of Hamiltonian (2) at $\mathbf{h} \| O Z$ will be provided, using selfconsistent field approximation that corresponds to the spin fluctuation neglecting and to the change of average of spin operators products at different sites by product of averages. In this case the ground state energy $E_{\text {gr }}$, normalizing on one cell (for nearest both inter- and intrasublattice different spins) is equal to:

$$
E_{\mathrm{gr}}=\frac{1}{2} \sum_{\alpha \beta} J_{\alpha \beta} z_{\alpha \beta} \mathbf{s}_{\alpha} \mathbf{s}_{\beta}+\frac{1}{2} \sum_{\alpha \beta} J_{\alpha \beta}^{Z} z_{\alpha \beta} s_{\alpha}^{Z} s_{\beta}^{Z}+D \sum_{\alpha} Q_{\alpha}^{Z Z}-h_{\|} \sum_{\alpha} s_{\alpha}^{Z},
$$

where $\mathbf{s}_{\alpha}$ is the quantum-mechanical average of the spin of the $\alpha$-th sublattice in the ion ground state; $z_{\alpha \beta}$ is the number of the nearest neighbors from the same $\left(z_{\alpha \alpha}\right)$ and another $\left(z_{\alpha \beta} \equiv z_{12}\right)$ sublattices. Introduced also are the averages for components of spin quadrupole moment $Q_{\alpha}^{Z Z}=$ $\left\langle\psi_{\alpha}^{(0)}\left|\left(S_{\mathbf{n} \alpha}^{Z}\right)^{2}\right| \psi_{\alpha}^{(0)}\right\rangle$ [38-40], where $\psi_{\alpha}^{(0)}$ is the wave function of the ground state of the ion from $\alpha$-sublattice. Note that for AFM the intersublattice exchange is $J_{12} z_{12} \equiv I>0$. At the same time, the value $J_{11} z_{11}=J_{22} z_{22} \equiv J$ of intrasublattice exchange can be of any sign, which is assumed for 
simplicity as furthering to the ordering, $J<0$. The exchange anisotropy, in this case, satisfies the conditions of its "easy-plane" type: $J_{12}^{Z} z_{12} \equiv \triangle I<0$ and $J_{11}^{Z} z_{11}=J_{22}^{Z} z_{22} \equiv \triangle J>0$.

Let us impose for spins of each sublattice their proper (rotating) coordinate systems $\xi_{\alpha} \eta_{\alpha} \zeta_{\alpha}$ such, that $\alpha$ th sublattice average spin is always oriented along $\zeta_{\alpha}$ axis, what means that this axis is the quantization one for this spin sublattice, and $\xi_{\alpha}$ axis is lain in $Z \zeta_{\alpha}$ plane. Then the correct wave function of the ground spin state of the $\alpha$-th sublattice in such a coordinate system, as it is well known, will have the following form ${ }^{2}[37]$ :

$$
\psi_{\alpha}^{(0)}=\cos \phi_{\alpha}|1\rangle+\sin \phi_{\alpha}|-1\rangle
$$

where $| \pm 1\rangle$ and $|0\rangle$ are eigenfunctions of operator $S_{\mathbf{n}_{\alpha}}^{\zeta}$ in bracket representation. Next, it can be calculated, using (4) the quantum-mechanical spin and quadrupole averages:

$$
s=\cos 2 \phi, \quad Q^{\zeta \zeta}=1, \quad Q^{\xi \xi}=\frac{1}{2}(1+\sin 2 \phi), \quad Q^{\eta \eta}=\frac{1}{2}(1-\sin 2 \phi) .
$$

Sublattice indices are omitted in the expressions (5), because, as already noted, with the chosen field direction, the evident dependence of observables on index $\alpha$ is absent.

The use of functions (4) makes it possible to obtain the energy (3) at $\mathbf{h} \| O Z$ as follows:

$$
\begin{aligned}
E_{\mathrm{gr}}= & I \cos ^{2} 2 \phi \cos 2 \theta-|J| \cos ^{2} 2 \phi+J_{Z} \cos ^{2} 2 \phi \cos ^{2} \theta+2 D\left[\cos ^{2} \theta+\frac{\sin ^{2} \theta}{2}(1+\sin 2 \phi)\right] \\
& -2 h_{\|} \cos \theta \cos 2 \phi,
\end{aligned}
$$

where $J_{Z} \equiv \triangle J-\triangle I$ and $\theta$ is the angle between the sublattice magnetization and $O Z$ axis.

The field behavior of mean spin (and its direction) for each sublattice in the field should be found in order to determine magnetization, magnetic susceptibility and subsequently striction. The same calculations should also be made for spin quadrupole moment. As reported in references $[38,39]$, solution of the problem of spin configuration in the magnetic field suggests minimization of expression (6) by all available unknown quantities: the geometric angle $\theta$ and (see equation (4)) the angle $\phi$ of quantum states mixture. This method of finding the observables, being completely an equivalent to the solution of quantum self-consistent problem, is more convenient and more consistent, because it allows to perform generalization on the case of finite temperatures $[27,28]$.

The equations for both required angles are:

$$
\begin{aligned}
& \frac{\partial E_{\mathrm{gr}}}{\partial \phi}=-2\left(I \cos 2 \theta-|J|+J_{Z} \cos ^{2} \theta\right) \sin 4 \phi+2 D \sin ^{2} \theta \cos 2 \phi+4 h_{\|} \cos \theta \sin 2 \phi=0, \\
& \frac{\partial E_{\mathrm{gr}}}{\partial \theta}=-\left(2 I+J_{Z}\right) \sin 2 \theta \cos ^{2} 2 \phi-D \sin 2 \theta(1-\sin 2 \phi)+2 h_{\|} \sin \theta \cos 2 \phi=0 .
\end{aligned}
$$

As it is known from reference [36], the set of equations (7) and (8) has two solutions for the case of the absence of the external magnetic field: non-magnetic one, $s=0$, that exists at $D>2(I+|J|)$ and "magnetic" one at $D \leqslant 2(I+|J|)$, with which the reduced value of single-site mean spin

$$
s=\sqrt{1-\frac{D^{2}}{4(I+|J|)^{2}}}<1
$$

is associated.

The initial ground state of the system should be the singlet state, $s=0$, so that the quantum phase transition (at the magnetic field $\mathbf{h} \| O Z$ ) from this state to magnetically ordered state occurs. So, assume that the above explicit inequality, i.e. $D>2(I+|J|)$, is satisfied. With this ratio of the model parameters, the ground state of the system is really nonmagnetic, and the ordering in the absence of magnetic field cannot be realized at any temperatures [39]. In other words, ratio (9)

\footnotetext{
${ }^{2}$ It should be denoted, that ground state functions, given in [20] for laboratory (crystallographic) coordinates system, can not be considered as eigen ones, until the self-consistent problem is solved (see [38]).
} 
determines the condition of singletness of magnet ground state, which is Van Vleck state. Solution $s=0$ is satisfied in the interval $h_{\|}<h_{s}=\sqrt{1-(I+|J| / D)}$.

As the field grows, the finite value, $s \neq 0$, of the mean spin on the site appears. It can be derived from equation (8) that the expression for the average spin orientation relatively to the crystallographic axis is:

$$
\cos \theta=\frac{h_{\|} \cos 2 \phi}{D(1-\sin 2 \phi)+\left(2 I+J_{Z}\right) \cos ^{2} 2 \phi} .
$$

From equations (7) and (10) it can be seen that in large fields, where $h_{\|} \geqq h_{\text {flip }}$ (where $h_{\text {flip }} \equiv$ $\left.D+2 I+J_{Z}\right)$ the state, in which the spins of both sublattices are directed along "hard" $(\theta=0)$ axis, is established. Then, spin projection on the field direction will be maximum and equal to $s=S=1$. For $h_{\|}<h_{\text {flip }}$, the spins of sublattices are orientated at a finite angle $0<\theta \leqslant \pi / 2$ to the "hard" axis.

\section{Thermodynamic analysis}

Using formulas (5) and substituting (10) into the equation (6), the ground state energy in the form of functional can be obtained:

$$
E_{\mathrm{gr}}=-(I+|J|) s^{2}+D\left(1-\sqrt{1-s^{2}}\right)-\frac{h^{2} s^{2}}{D\left(1+\sqrt{1-s^{2}}\right)+\left(2 I+J_{Z}\right) s^{2}},
$$

which depends on the spin polarization s only. The expansion of this energy over the small $s$ gives:

$$
E_{\mathrm{gr}}=\frac{h_{s}}{D}\left(h_{s}-h_{\|}\right) s^{2}+\frac{D}{8}\left(1+\frac{2 h_{s}^{2}\left(2 I+J_{Z}\right)}{D^{3}}\right) s^{4},
$$

where $h_{s}=D \sqrt{1-2(I+|J|) / D}$ is the critical field of the appearance of magnetization.

In the expansion (12), which refers to the field region $h_{\|} \rightarrow h_{s}$, one can restrict to the terms that are not higher than of 4 th power by $s$. Actually this expansion for the ground state energy is similar to the free energy expression in Landau theory of phase transitions. However, in equation (12) the ground state spin polarization corresponds to the order parameter, and the leading value, that results in the phase transition, is not the temperature, but the magnetic field. It can be also seen from equation (12) that at $h_{\|}<h_{s}$ the coefficients at $s^{2}$ and $s^{4}$ are positive, and so the ground state of spin system will be Van Vleck non-magnetic single-ion state. At the point $h_{\|}=h_{s}$ the sign of coefficient at $s^{2}$ changes, and in the fields $h_{\|}>h_{s}$ the spin polarization (of still nondegenerate ground state) spontaneously appears. The value of polarization can be readily found by minimization of $E_{\mathrm{gr}}(12)$ :

$$
\frac{\partial E_{\mathrm{gr}}}{\partial s}=2 s\left[\frac{h_{s}}{D}\left(h_{s}-h_{\|}\right)+\frac{D}{4}\left(1+\frac{2 h_{s}^{2}\left(2 I+J_{Z}\right)}{D^{3}}\right) s^{2}\right]=0 .
$$

From equation (13) it follows that near the quantum critical point $h_{\|} \geqslant h_{s}$ this polarization (or simply the ground state spin)

$$
s\left(h_{\|}\right)=\sqrt{\frac{4 h_{s}\left(h_{\|}-h_{s}\right)}{D^{2}+2 h_{s}^{2}\left(2 I+J_{Z}\right) / D}}
$$

fundamentally depends on the field.

In the same vicinity, $h_{\|} \geqslant h_{s}$, of the quantum critical point, the angle $\theta$ between vector $\mathbf{s}$ and axis $O Z$ is determined by the expression:

$$
\cos \theta=\frac{h_{s}}{2 D} \sqrt{\frac{4 h_{s}\left(h_{\|}-h_{s}\right)}{D^{2}+2 h_{s}^{2}\left(2 I+J_{Z}\right) / D}} .
$$


Thus, it is found, that at $h_{\|}=h_{s}$ the spin polarization spontaneously arises as field grows in the very "easy" plane, because at $h_{\|}-h_{s} \rightarrow 0$ the angle $\theta \rightarrow \pi / 2$. In other words, it turns out that at the moment of its appearance, vector $\mathbf{s}\left(h_{\|} \geqslant h_{s}\right)$ is perpendicular to the longitudinal field: $\mathbf{s} \perp \mathbf{H} \| O Z$. Further magnetic field growth leads not only to the decrease of $\theta$, as it follows from equations $(14-15)$, but also to a simultaneous increase of spin polarization, that is the bigger its value is, the more it flattens against the "hard" axis.

On the whole, the induced tilt of the magnetic sublattices, and thereafter the magnetization of SAFM includes two processes: the classical rotation of spins (sublattice magnetizations) and purely quantum (due to the change of the angle $\phi$ ) growth of single-site polarization $s\left(h_{\|}\right)$. Both processes also take place at $T=0$. The AFM magnetization (normalizing on one magnetic atom) is described by the evident product:

$$
m_{\|} \equiv m\left(h_{\|}\right)=s\left(h_{\|}\right) \cos \theta=\frac{2 h_{s}^{2}\left(h_{\|}-h_{s}\right)}{D^{3}+2 h_{s}^{2}\left(2 I+J_{Z}\right)} .
$$

As a result, one arrives to an unexpected result: the observed magnetization near the critical field of quantum transition from singlet to spin-polarized state depends linearly - as in classical AFMs - upon the external magnetic field, that induces the vary transition. From this comes another rather remarkable conclusion: at such a phase transition the magnetic susceptibility of a system should have a jump.

Thus, in the framework of the approach that is similar to the Landau thermodynamic approach, it was demonstrated that the spin polarization is the only order parameter for quantum phase transition from Van Vleck phase to the AFM phase induced by magnetic field $\mathbf{h} \| O Z$. Despite the fact, that calculations were made for the case $T=0$, the required polarization proves to be essentially dependent on the external field. It should be reminded, that in classical AFMs, ion spin polarization is fixed at $T=0$ and it is not evaluated in the field, while in Van Vleck system it appears as a consequence (in terminology of reference [24]) of quantum phase transition [27,28].

Next, attention should be drawn to the following analogy, that SIA, reducing the average spin, plays a role of "disordering" factor, and in this sense it can be compared with entropy. It (SIA) leads to the mixture (or linear combination) of quantum states that results in the absence of spin polarization of ions in their ground state. Exchange and magnetic fields, on the contrary, resist this, "magnetizing" the system and causing a spontaneous (or forced) spin polarization, which, at the moment of its appearance, is directed perpendicularly to the magnetic field.

The studied quantum phase transition between Van Vleck (also ordered, inessential) and AFM states is, as it was seen, the consequence of competition of different interactions (exchange, Zeeman and spin-orbital, that lies at the heart of SIA). Therefore, such a quantum transformation can be naturally identified as the magnetic phase transition of "displacement" but not of "order-disorder" type. As distinct from the last one, the transition of displacement type is not the transition in the system of spins, which fluctuates "up" and "down" between degenerated (or almost degenerated) quantum states, because the ground state of quantum paramagnets is always non-degenerated and its polarization is the direct consequence of rearrangement of this state in the external field.

Note that the applicability of phenomenological theory, which is based on the expansion (11) is confined by the fields $h_{\|} \geqslant h_{s}$ in the vicinity of critical point $h_{s}$. In the field region $h_{\|} \gg h_{s}$ the magnetization process should be analyzed with more exact expressions both for ground state energy and for the equations that define the spin configurations. However, the latter can be easily found numerically.

\section{The magnetization and magnetic susceptibility}

Substituting expression (5) into equation (11), the following equation, which describes the spin polarization as the function of longitudinal field, can be obtained:

$$
s\left(D-2(I+|J|) \sqrt{1-s^{2}}-\frac{D\left(1+\sqrt{1-s^{2}}\right) h_{\|}^{2}}{\left(D\left(1+\sqrt{1-s^{2}}\right)+\left(2 I+J_{Z}\right) s^{2}\right)^{2}}\right)=0 .
$$


Note that this equation refers both to the fields $h_{\|}<h_{s}$ of the existence of Van Vleck phase, where (see $(12)) h_{s}=\sqrt{D^{2}-2 D(I+|J|)}$, the nonmagnetic, $s=0$, state is stable, and to the region $h_{s} \leqslant h_{\|} \leqslant h_{\text {flip }}$ of the AFM phase (up to the point $h_{\text {flip }}$ of its flipping). It is obvious, that at the point (see (10) ) $h_{\|}=h_{\text {flip }}$, which corresponds to $\theta=0$, the polarization arrives at its maximum value $s=1$ on the site.

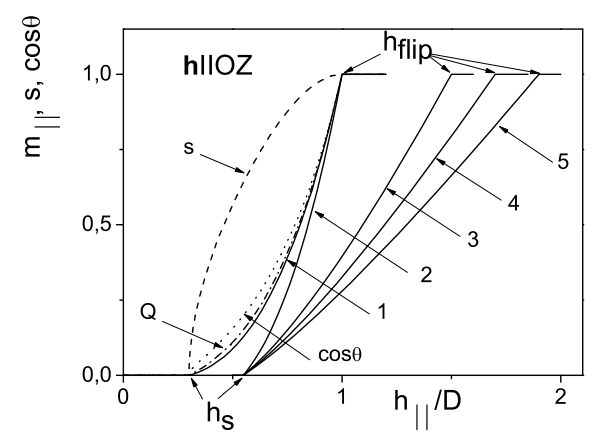

Figure 1. Magnetization $m_{\|}$(solid curves), spin $s$, quadrupole moment $Q$ and value of $\cos \theta$ versus field. The curves for $s, Q$ and $\cos \theta$ are calculated at $|J| / D=0.455$ and at condition $I=J_{Z}=0$. Magnetization curves 1-5 are for the following parameters: curve 1 is for $|J| / D=$ $0.455, I=J_{Z}=0$; curve 2 is for $|J| / D=0.35, I=J_{Z}=0$; curve 3 is for $|J| / D=0.35$, $I=0, J_{Z} / D=0.5$; curve 4 is for $|J| / D=0, I / D=0.35, J_{Z}=0$; curve 5 is for $|J| / D=0.15$, $I / D=0.2, J_{Z} / D=0.5$.

Using equation (17), the behavior $s\left(h_{\|}\right)$in the region $h_{s} \leqslant h_{\|} \leqslant h_{\text {flip }}$ can be found, and from equation (10) - the angle $\theta$ can also be found. Then, it is not difficult to define the field dependence of the quadrupole $Q_{\alpha}^{Z Z}$ in equation (3). In the framework of such an approach, the field dependencies of magnetization were calculated (see figure 1).

Curve 1 in figure 1 was plotted for the case where intrasublattice exchange prevails, while intersubalttice and anisotropic ones are omitted. At chosen parameters, the magnetic sublattices are fixed artificially, because this extreme case actually corresponds to the two independent AFMs, The field behavior for $s, Q_{\alpha}^{Z Z} \equiv Q$ and $\cos \theta$ is also shown in figure 1 for these parameters. It can be seen that at the point $h_{\|}=h_{s}$ the average site spin really spontaneously appears and exists in the region $h_{\|}>h_{s}$. With further field growth, the value of $s$ is increasing, guided by velocity of the change of angle $\theta$. This velocity, however, becomes higher, when the field approaches the flipping field and, correspondingly, $s \rightarrow 1$. In such a case the intrasublattice exchange (due to its isotropy) has no effect on spin saturation, and so the value $h_{\text {flip }}$ of this critical field is completely defined by the single-ion anisotropy.

From curve 1 , that refers to the case $D-2|J| \ll D$, it follows, that the fields $h_{\text {flip }}$ and $h_{s}$ differ quite weakly $\left(h_{\mathrm{flip}} / h_{s} \approx 3\right)$, although in the experiment $[22,23]$ their ratio reaches 6 , and from the data of the study [30] this ratio is about 8. Besides, the field dependence of magnetization for the considered case $I=0$ reveals, as can be seen, large nonlinearity, while the experimental data for all the above mentioned compounds are evidence of nearly linear dependence of magnetization on magnetizing force.

It should be noted that the case, in which inequality $(D-2|J|) / D \ll 1$ satisfies, is physically available, but it cannot be justified from the experimental point of view. To demonstrate this in figure 1, the curve 2 is plotted, for which the difference between parameters in intrasublattice exchange and SIA is chosen not less, but bigger than for curve 1 . This choice really leads to the increase of the field $h_{s}$ and results in the decrease of the ratio $h_{\text {flip }} / h_{s}$, which indicates that attempting to interpret the experimental magnetization, the intersublattice exchange cannot be neglected.

It is interesting, that when $I / D \rightarrow 0$, equation (17) has an exact solution, using which the 
ground state energy can be written in the form of a function of magnetic field:

$$
E_{\mathrm{gr}}=\frac{1}{4 D^{2}|J|}\left(h_{\|}^{2}-h_{s}^{2}\right)^{2} .
$$

Then the magnetization (normalizing on one magnetic ion again) takes the following form:

$$
m_{\|}=-\frac{\partial E_{\mathrm{gr}}}{\partial h_{\|}}=\frac{h_{\|}}{2 D^{2}|J|}\left(h_{\|}^{2}-h_{s}^{2}\right) .
$$

The last dependence is described by curves 1 and 2 in figure 1 . From equations (18) and (19), quite a big field nonlinearity of magnetization in the antiferromagnetic phase can bee seen. Expression (19), for fields $h_{\|} \rightarrow h_{s}$ can be also presented in the form of equation (16), when $2|J| \rightarrow D$.

Now let us consider the opposite limiting case, when intersublattice exchange is the biggest. It can be seen that even if one preserves the exchange (that formally gives the same value of $h_{s}$ ), which effects the spin from another sublattice, the change of magnetization (curve 4 in figure 1) occurs. The AFM (intersublattice) exchange, unlike the intrasublattice one, leads to the growth of the field $h_{\text {flip }}$, because in this case the external field will overcome the effect of the same anisotropy, on the one hand, and the effect of exchange field that prevents parallel orientation of sublattice spins, on the other hand.

Curve 3 already shows the nonlinearity decrease in $m\left(h_{\|}\right)$, as if it was rectified by intersublattice exchange (or by its anisotropic part). At the same time, AFM exchange together with the external magnetic field (in the region $h_{\|}>h_{s}$ ), while resisting the anisotropy, leads to the establishment of spontaneous polarization. In the large fields, when polarization tends to its maximum value, the behavior of exchange in SAFM does not differ from that in classical AFMs: it simply resists the parallel configuration of both sublattice spins.

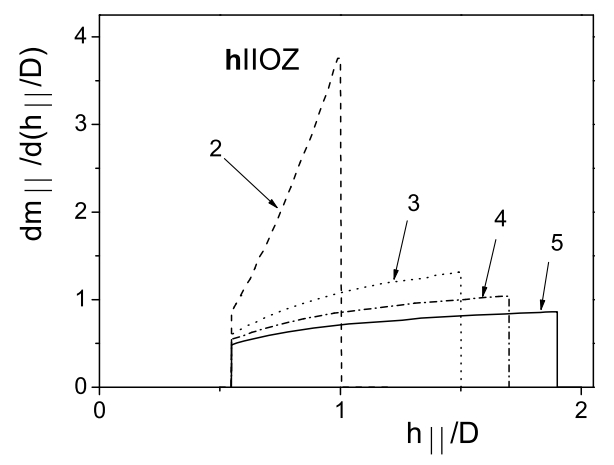

Figure 2. Magnetic susceptibility $\chi_{\|}$versus field. The numbers of the curves correspond with parameters, that were used in plotting the lines with the same numbers as in figure 1.

Curves 3 and 5 demonstrate the effect of easy-plane exchange anisotropy. Actually this anisotropy does not change the position of critical field $h_{s}$, but it also does not "desire" the establishment of collinear state, when $\mathbf{s}_{1} \rightarrow \mathbf{s}_{2} \| O Z$. At the same time the account of exchange anisotropy of easy-plane type being taken into account, it permits to obtain such a behavior of magnetization that is close to linear and is observed in the studies $[22,23,29,30]$. For clarifying how well the linear dependence corresponds to $m\left(h_{\|}\right)$, the magnetic susceptibility $\chi_{\|}=\mathrm{d} m_{\|} / \mathrm{d} h$ is shown in figure 2 for the same parameters as in figure 1.

Since magnetization is nothing more but $m_{\|}=s \cos \theta$, where $s=s\left(h_{\|}\right)$, the longitudinal magnetic susceptibility of SAFMs is naturally to be represented in the form of the two above 
mentioned terms, i.e., the classical $\chi_{\mathrm{cl}}$ and the quantum $\chi_{\text {quan }}$, so that

$$
\chi_{\|}=\chi_{\mathrm{cl}}+\chi_{\text {quan }}, \quad \chi_{\mathrm{cl}}=s \sin \theta \frac{\partial \theta}{\partial h}, \quad \chi_{\text {quan }}=\cos \theta \frac{\partial s}{\partial h} .
$$

As can be seen from figure 1 , near $h_{s}$ the biggest growth reveals the spin polarization $s$, so in the fields $h_{\|} \rightarrow h_{s}$ the "quantum" contribution will dominate in the magnetic susceptibility. And when the value of spin polarization saturates $\left(s\left(h_{\|} \rightarrow h_{\text {flip }}\right) \rightarrow 1\right)$, the susceptibility will be mainly controlled by classical term (see equations $(20)$ ).

Figure 2 shows that, when intrasublattice exchange is really the largest, then magnetic susceptibility grows, increasing in the field region $h_{s} \leqslant h_{\|} \leqslant h_{\text {flip }}$ four times. If intersublattice exchange and/or exchange anisotropy "switches" on, then field dependence of differential magnetic susceptibility $\chi_{\|} \equiv \chi\left(h_{\|}\right)$becomes essentially weaker. Nevertheless, it can be seen from the curves, shown in figure 1 that nonlinearity of function $m\left(h_{\|}\right)$in the antiferromagnetic phase at the chosen parameters remains quite noticeable. The case, when within the boundaries of this phase, the value

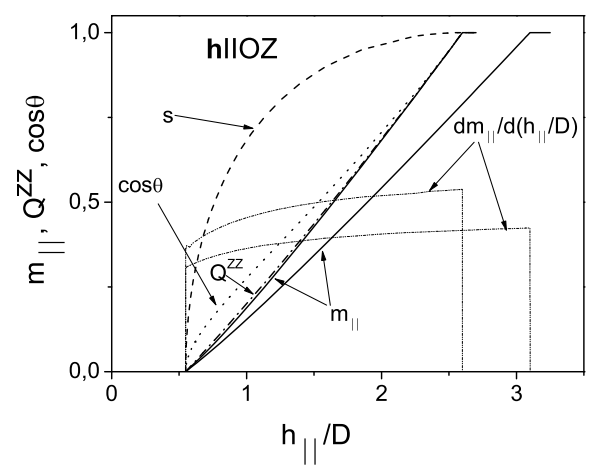

Figure 3. Longitudinal magnetization $m_{\|}$and magnetic susceptibility $\chi_{\|}$versus field at $|J| / D=$ $0.05, I / D=0.3, J_{Z} / D=1$ and 1.5. The functions $s\left(h_{\|}\right), \cos \theta\left(h_{\|}\right)$and $Q\left(h_{\|}\right)$are shown only for $|J| / D=0.05, I / D=0.3, J_{Z} / D=1$.

of $\chi\left(h_{\|}\right)$changes $(30-50 \%)$, is shown in figure 3 , which meets the model parameters $|J| / D=0.05$, $I / D=0.3, J_{Z} / D=1$ or $J_{Z} / D=1.5$. In other words, the exchange anisotropy is comparable or even exceeds the SIA. At such ratios between the parameters, the field $h_{\text {flip }}$ almost five times exceeds the field $h_{s}$ (one should note that the experimentally observed ratio is $h_{\text {flip }} / h_{s} \approx 6[22,23]$ ).

In the same figure 3 the dependencies of $s\left(h_{\|}\right), \cos \theta\left(h_{\|}\right)$and $Q\left(h_{\|}\right)$are shown for parameters $|J| / D=0.05, I / D=0.3$ and $J_{Z} / D=1$. The behavior of $Q\left(h_{\|}\right)$almost coincides (see figure 1 ) with the field dependence $m\left(h_{\|}\right)$. Moreover, it follows from figure 3 that exchange anisotropy, even comparable with SIA, does not fully linearize the function $m\left(h_{\|}\right)$. As it was mentioned, this fact can be explained by the existence of two different regions in the magnetization of SAFM.

In the first of these regions, near $h_{s}$, the quantum process, as it was pointed out, is determinant and the magnetization is defined basically by the appearance and growth of $s\left(h_{\|}\right)$. In the second region, in the vicinity of $h_{\|} \leqslant h_{\text {flip }}$, the classical rotation of sublattice spins towards the field direction becomes more important, at essentially less (but still present) role the vary spin value change plays. It is obvious that in this region, the susceptibility depends much weaker on the value of magnetic field. So, it can be supposed, that the flipping of tilted AFM sublattices, or the transition of Van Vleck system between the induced two- and one-sublattice magnetically ordered states occurs as an orientation quantum phase transition in ordinary AFM, when the variation of sublattice magnetization directions is in fact the only process taking place.

However, even for this field transition, the quasiclassical approach does not give the correct solution to $m\left(h_{\|}\right)$in spin nematic. Indeed, one could suppose that near the flipping field, when 
$s\left(h_{\|} \rightarrow h_{\text {flip }}\right) \approx 1$, the quasiclassical magnetic energy in the ground state takes the form:

$$
E_{\mathrm{gr}}=I \cos 2 \theta+\left(J_{Z}+D\right) \cos ^{2} \theta-2 h_{\|} \cos \theta=0 .
$$

Then, from equation (21) there immediately follows the equation

$$
\frac{\mathrm{d} E_{\mathrm{gr}}}{\mathrm{d} h_{\|}}=2\left[-\left(2 I+J_{Z}+D\right) \cos \theta+h_{\|}\right] \sin \theta=0,
$$

which shows that in the vicinity of $h_{\|} \rightarrow h_{\text {flip }}$ the magnetization of the tilted $(\theta \neq 0)$ phase is proportional to the field: $m_{\|}=\tilde{\chi}_{\|} h_{\|}$, where

$$
\tilde{\chi}_{\|}=\frac{1}{D+2 I+J_{Z}} \equiv \frac{1}{h_{\text {flip }}}=\text { const. }
$$

It can be seen that in the field $h_{\text {flip }}$, the magnetization (on one spin) is $m_{\|}=1$. However, the susceptibility (23) differs from the exact ratio (20) and gives physically incorrect behavior of magnetization. It is connected with the fact, that in the region $h_{\|} \rightarrow h_{\text {flip }}$ it appears that $m_{\|}$ depends linearly on the magnetic field, and asymptotically tends to zero at $h_{\|} \rightarrow 0$. As for the plots, shown in figures 1 and 3 , it is easy to see that function $m\left(h_{\|}\right)$, although it behaves linearly by field, but nevertheless it depends on the magnetic field in an indirect proportion.

An important conclusion follows from this: the quasiclassical approach (21), based on the substitution of quadrupole moment $Q^{Z Z}$ by the average spin $Z$ th projection square, appears to be unapplicable even in such a field region, where spin polarization almost reaches its saturation value $s \rightarrow 1$.

\section{The magnetization in transversal magnetic field}

As it was mentioned, the phase transition to the AFM state does not occur at $\mathbf{h} \perp O Z$, although the magnetic field magnetizes the system.

Let us suppose that due to AFM exchange in the easy plane, two sublattices are formed. Then, their spins lie in this plane and are identically tilted to the field. In this case, the ground state energy is:

$$
E_{\mathrm{gr}}=I \cos 2 \varphi \cos ^{2} 2 \phi-|J| \cos ^{2} 2 \phi+D(1+\sin 2 \phi)-2 h_{\perp} \cos \varphi \cos 2 \phi,
$$

where $\varphi$ is the angle between vector $\mathbf{s}_{1}$ (or vector $\mathbf{s}_{2}$ ) and field $\mathbf{h}$, and the angle between $\mathbf{s}_{1}$ and $\mathbf{s}_{2}$ is twice larger, $2 \varphi$.

The spin configuration will be defined, as usual, by minimizing the energy (24). As a result, it is the set of equations (compare equations (7) and (8)):

$$
\begin{aligned}
& \frac{\partial E_{\mathrm{gr}}}{\partial \varphi}=-2 I \cos ^{2} 2 \phi \sin 2 \varphi+2 h_{\perp} \sin \varphi \cos 2 \phi \\
& \frac{\partial E_{\mathrm{gr}}}{\partial \phi}=-2(I \cos 2 \phi-|J|) \sin 4 \phi+2 D \cos 2 \phi+4 h_{\perp} \cos \varphi \sin 2 \phi=0 .
\end{aligned}
$$

Equation (25) has two solutions. For the first of them, $\varphi=0$ and it corresponds to onesublattice magnetization, when the polarization of magnetic ions is directed along the field. The second solution $\cos \varphi=h_{\perp} /(2 I \cos 2 \phi)$ provides the existence of two sublattices. The latter, taking into account equation (5), can be rewritten in the usual form:

$$
\cos \varphi=h_{\perp} / 2 I s
$$

The denominator of equation (27) is the intersublattice exchange field, and this expression is similar to the expression for the magnetic sublattice tilt angle in classical AFMs [2,3]. Nevertheless, it should be noted that the spin in equation (27) is not equal to its maximum value. 
Substituting (27) in (26), one arrives at the equation:

$$
2[2(I+|J|) \sin 2 \phi+D] \cos 2 \phi=0 .
$$

It follows from equation (28) that spin polarization for AFM state at $\mathbf{h} \perp O Z$ should be equal to (9). However, the model parameters, accepted above, are such that the denominator under the root in equation (9) is larger than 1, and non-polarized singlet is the ground state of ions. Thus, the solution (27) is possible only for initially polarized ground state, or when the AFM (not singlet) phase is realized in the system even at $h_{\perp}=0$. But if without field the polarization is $s=0$, then from the set of equations (25)-(26) a fundamentally different result follows: the critical field of polarization appearance in the transversal geometry is the $h_{\perp}=0$. The distinction from "longitudinal" case, for which the critical field is finite, is easy to explain. At any fields $h_{\perp} \neq 0$ the ground state with $S_{Z}=0$ (in crystal coordinate system) is immediately admixed with the ionic exited states, which have $S_{Z}= \pm 1$. In the case of longitudinal field, there is a threshold for such an admixture. Then, taking into account that the transversal field does not induce AFM phase, one can obtain:

$$
E_{\mathrm{gr}}=(I+|J|) s^{2}+D\left(1-\sqrt{1-s^{2}}\right)-2 h_{\perp} s .
$$

Note that at $\mathbf{h} \perp O Z$ the spin polarization is always equal to magnetization, which is directed along $\mathbf{h}$, i.e. $m_{\perp}=s$. Minimizing energy (29), one readily arrives at the equation

$$
\frac{\partial E_{\mathrm{gr}}}{\partial s}=2(I+|J|) s+D \frac{s}{\sqrt{1-s^{2}}}-2 h_{\perp}=0
$$

which permits to define the dependence of spin polarization upon the transversal field.

The field behavior for $m_{\perp}$, which is obtained from equation (30) is shown in figure 5 . It is seen that if intrasublattice exchange dominates in the system, then the magnetization increases rapidly, and if intersublattice exchange is "added", then the magnetization slows down.

Despite the fact that at $\mathbf{h} \perp O Z$ the average spins are also oriented perpendicularly to $Z$, spin quadrupole moment $Q^{Z Z}$ versus field reveals the behavior (see figure 5) similar to the spin polarization. Magnetization rate decreases as the field grows and the magnetic susceptibility has a maximum at $h_{\perp} \rightarrow 0$. The normalized magnetic susceptibility $\chi_{\perp}^{(0)}=\chi\left(h_{\perp}=0\right)$ is also plotted in figure 4.

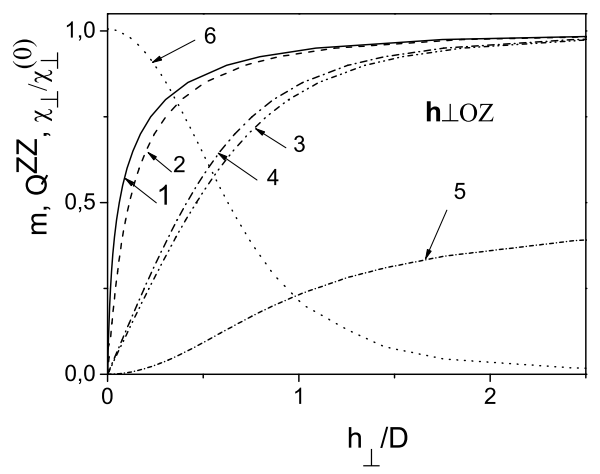

Figure 4. Field behavior of $m\left(h_{\perp}\right)$ (lines 1-4), $Q^{Z Z}\left(h_{\perp}\right)$ (line 5) and $\chi_{\perp}$ for $\mathbf{h} \perp O Z$. The line 1 is calculated for parameters $|J| / D=0.455, I=0$, the line 2 is for $|J| / D=0.35, I=0$, the line 3 is for $|J| / D=0, I=0.35$, lines $4-6$ are for $|J| / D=0.05, I=0.3$.

Using equation (30), the expression for magnetic susceptibility at $\mathbf{h} \perp O Z$ can be obtained; it has the form:

$$
\chi_{\perp} \equiv \chi\left(h_{\perp}\right)=\frac{1}{2(I+|J|)+D\left(1-s^{2}\right)^{-3 / 2}} .
$$


It is easy to see that in large fields, when $s \rightarrow 1$, transversal susceptibility $\chi_{\perp} \rightarrow 0$. In the opposite limit $h_{\perp} \rightarrow 0$, the magnetic susceptibility is equal to:

$$
\chi_{\perp}^{(0)}=\frac{2}{D+2(I+|J|)} .
$$

It also follows from equation (32), that when the intrasublattice exchange increases, the value of $\chi_{\perp}^{(0)}$ grows and, on the contrary, at the increase of intersublattice exchange it decreases. This is a usual situation in physics of phase transitions, because the growth of intrasublattice exchange at $I=0$ can result in ferromagnetic state with susceptibility singularity characteristic of such type of transition (it goes to the infinity at the transition point). At the same time, the transition to the AFM state is not accompanied by the abnormal growth of magnetic susceptibility. Indeed, the point of phase transition to the AFM phase corresponds to the equality $D=2(I+|J|)$. At its substitution in equation (32) the value $\chi_{\perp}^{(0)}=1 / 2 I$ is directly obtained. The same will be the value of magnetic susceptibility in the AFM phase, the magnetization of which is determined by the expression (27).

\section{Conclusion}

Thus, it was obtained that the phase transition in SAFM to magnetically ordered state, induced by magnetic field, is the quantum phase transition. The spin polarization of the magnetic ion ground state is the order parameter of this phase transition. The Landau theory can be used for its description. The considered transition is a consequence of the competition of different interactions, and, what is important, it appears in the field that is perpendicular to the easy plane. Such a field does not reduce the symmetry in this plane, leaving all directions in it equivalent. Conservation of degeneracy for directions of sublattice magnetizations in the "easy" plane is the crucial symmetrical condition for the phase transition to the AFM state with spontaneous magnetizations lying in this plane.

Also, it is shown that in the magnetic field induced AFM phase, the spin polarization (magnetization) of the sublattice changes continuously from zero, reaching its maximum at the spin flipping point. In contrast to classical Neel AFM, in the magnetic phase of Van Vleck (singlet) AFM, the value of the sublattice magnetization strongly depends on the field. The same field dependence has an angle that defines the deviation of sublattice magnetization from the field direction. At the same time, the magnetization of a system as a whole, being weekly dependent on the field, shows almost linear field behavior (allowing the exchange anisotropy).

Finally, it is necessary to make a methodical remark. The above results were obtained in an approximation of self-consistent field. It was assumed that more accurate calculations would not give any qualitative results. However, they might have a quantitative effect. A separate paper will be devoted to the quantitative comparison of calculations and the available experimental data.

We are grateful to Prof. S.M. Ryabchenko, who drew our attention to experimental studies $[22,23]$, as well as to the problem of magnetostriction in singlet magnets.

This work is partially supported by Grant of Ministry of Education and Science of Ukraine (No. 25.2/043).

\section{References}

1. Sachdev S. Quantum phase transitions. Cambridge Univ. Press, Cambridge 1999.

2. Neel L. Magnetisme et champ molekulaire local. - In Conference Nobel, 11 decembere 1970. Reimpression de Les prix Nobel en 1970, Stockholm, 57.

3. Borovik-Romanov A.S. Antiferromagnetism. Progress in Science. AN USSR, Moscow, 1962 (in Russian).

4. Turov E.A. Physical Properties off Magnetically ordered Crystals. N.-Y.-London, Academic Press, 1965, 222.

5. Belov K.P., Zvezdin A.K., Kadomtseva A.M., Levitin R.Z. Orientational Transitions in Rare-Earth Magnets. Science, Moscow, 1979. 
6. Andreev A.F., Marchenko V.I., Sov. Phys. - Usp., 1980, 23, 37.

7. Wilkinson M.K., Cable J.W., Wollan E.O., Koehler W.C., Phys. Rev., 1959, 113, 497.

8. Lines M.E., Phys. Rev., 1963, 131, 546.

9. Leech J.W., Manuel A.J., Proc. Phys. Soc., London, Sec. B, 1956, 59, 210.

10. Starr C., Bitter F., Kaufmann A.R., Phys. Rev., 1940, 58, 977.

11. Billerey D., Terrier C., Pointon A.J., Redoules J.P., J. Magn. Magn. Mater., 1980, 21, 187.

12. Lozenko A.F., Parhomchuk P.E., Ryabchenko S.M., Trotsenko P.A., J. Low Temp. Phys., 1988, 14, 241.

13. Kalita V.M., Lozenko A.F., Ryabchenko S.M., Trotsenko P.A., Sov. Phys. - JETP, 2004, 99, 1054.

14. Kalita V.M., Lozenko A.F., Ryabchenko S.M., Trotsenko P.A., J. Low Temp. Phys., 2005, 31, 794.

15. Dorner B., Visser D., Stiegenberger U., Kakurai K., Steiner M., Z. Phys. B, 1988 72, 487.

16. Harrison A., Visser D., J. Phys.: Condens. Matter, 1992, 4, 6977.

17. Gekht R.S., Physics - Uspekhi, 1989, 32, 233.

18. Collins M.F., Petrenko O.A., Can. J. Phys., 1997, 75, 605.

19. Dumesh B.S., Physics - Uspekhi, 2000, 43, 365.

20. Zapf V.S., Zocco D., Hansen B.R., Jaime M., Harrison N., Batista C.D., Kenzelmann M., Niedermayer C., Lacerda A., Paduan-Filho A., Phys. Rev. Lett., 2006, 96, 077204.

21. Zvyagin S.A., Wosnitza J., Batista C.D., Tsukamoto M., Kawashima N., Krzystek J., Zapf V.S., Jaime M., Oliveira N.F., Jr., Paduan-Filho A., Phys. Rev. Lett., 2007, 98, 047205.

22. Zapf V.S., Corea V.F., Batista C.D., Murphy T., Palm E.D.,Jaime M., Tozer S., Lacerda A., PaduanFilho A., J. Appl. Phys., 2007, 101, 09E106.

23. Zapf V.S., Corea V.F., Sengupta P., Batista C.D., Tsukamoto M., Kawashima N., Egan P., Pantea C., Migliori A., Bets J.B., Jaime M., Paduan-Filho A. Preprint arXiv:cond-mat/0705.0365v1, 2007.

24. Stishov S.M., Physics - Uspekhi, 2004, 47, 789.

25. Pereverzev Yu.V., Borisenko V.G., Sov. Phys. Solid State, 1984, 26, 1249, (in Russian).

26. Rosenfeld Ye.V., JETP Lett., 1976, 24, 60 (in Russian).

27. Kalita V.M., Loktev V.M., Zh. Eksp. Theor. Fiz, 2004, 98, 1006.

28. Kalita V.M., Loktev V.M., Low Temp. Phys, 2006, 32, 114.

29. Haseda T., Wada N., Hata M., Amaya K., Physica B, 1991, 108, 841.

30. Tanaka Y., Tanaka H., Ono T., Oosawa A., Morishita K., Iio K., Kato T., Katori H.A., Bartashevich M.I., Goto T. Preprint arXiv:cond-mat/0104287, 2001.

31. Ivanov B.A., Khymyn R.S., Zh. Eksp. Theor. Fiz, 2007, 104, 307.

32. Nikuni T., Osikawa M., Oosawa A., Tanaka H., Phys. Rev. Lett., 2000, 84, 5868.

33. Matsumoto M., Normand B., Rice T.M., Sigrist M., Phys. Rev. Lett.,2003, 89, 077203.

34. Sherman E.Ya., Lemmens P., Busse B., Oosawa A., Tanaka H., Phys. Rev. Lett., 2003, 91, 057201.

35. Radu T., Wilheim H., Yushanhai V., Kovrizhin D., Goldea R., Tylczynski Z., Luhmann T., Steglich F., Phys. Rev. Lett., 2005, 95, 1272002.

36. Bugriy A.I., Loktev V.M., J. Low Temp. Phys., 2007, 33, 37.

37. Loktev V.M., Ostrovskiy V.S., J. Low Temp. Phys., 1994, 20, 775.

38. Kalita V.M., Ivanova I.M., Loktev V.M., J. Low Temp. Phys., 2002, 28, 475.

39. Kalita V.M., Loktev V.M., Physics - Solid State, 2003, 45, 1523.

40. Onufrieva F.P., Zh. Eksp. Theor. Fiz, 1985, 89, 2270 (in Russian).

41. Fridman Yu.A., Klevets Ph., Kozhemyako O.V., J. Magn. Magn. Mater., 2003, 264, 111.

42. Mitsai Yu.N., Fridman Yu.A., Kozhemyako O.V., Kosmachev O.A., J. Low Temp. Phys., 1999, 25, 569. 


\title{
Квантовий фазовий перехід: ван-флеківський антиферомагнетик у магнітному полі
}

\author{
І.М.Іванова ${ }^{1}$, В.М.Каліта ${ }^{2}$, В.О.Пашков ${ }^{3}$, В.М.Локтєв ${ }^{4}$ \\ 1 Національний технічний університет України “КПІ”, пр. Перемоги 37, Київ, 03056 Україна \\ 2 Інститут фізики НАН України, пр. Науки 46, Київ, 03680 Украина \\ 3 Національний авіаційний інститут, пр. Космонавта Комарова. 1, Київ, 03058 Україна \\ 4 Інститут теоретичної фізики ім. М.М. Боголюбова НАН України, вул. Метрологічна 14-б, Київ, 03143 \\ Україна
}

Отримано 7 травня 2008 р., в остаточному вигляді - 7 липня 2008 р.

\begin{abstract}
Проведено теоретичний опис індукованого магнітним полем квантового фазового переходу у антиферомагнітий стан у ван-флеківському антиферомагнетику з легкоплощинним типом однойонної анізотропії і величиною спінів магнітних йонів $S=1$. Показано, що при магнітному фазовому переході параметром порядку є величина спінової поляризації основного стану, а для опису цього переходу застосовна термодинамічна теорія фазових переходів Ландау. Вивчені магнітні властивості індукованої магнітним полем антиферомагнітної фази, проаналізовані польові залежності намагніченості і магнітної сприйнятливості. Зроблена спроба якісного порівняння отриманих результатів 3 наявними експериментальними даними.
\end{abstract}

Ключові слова: квантовий магнітний фазовий перехід, однойонна анізотропія, спінова поляризація, ван-флеківський антиферомагнетик

PACS: 75.10.-b, 75.10.Jm, 75.30.Gw, 75.30.Kz, 75.50.Ee 